\title{
Accelerator design for the Cornell High Energy Synchrotron Source upgrade
}

\author{
J. Shanks, J. Barley, S. Barrett, M. Billing, G. Codner, Y. Li, X. Liu, A. Lyndaker, \\ D. Rice, N. Rider, D. L. Rubin, A. Temnykh, and S. T. Wang \\ CLASSE, Cornell University, Ithaca, New York 14853, USA
}

(Received 17 October 2018; published 20 February 2019)

\begin{abstract}
During the summer and fall of 2018 the Cornell High Energy Synchrotron Source (CHESS) is undergoing an upgrade to increase high-energy flux for x-ray users. The upgrade requires replacing onesixth of the Cornell Electron Storage Ring (CESR), inverting the polarity of half of the CHESS beam lines, and switching to single-beam on-axis operation. The new sextant is comprised of six double-bend achromats (DBAs) with combined-function dipole-quadrupoles. Although the DBA design is widely utilized and well understood, the constraints for the CESR modifications make the CHESS-U lattice unique. This paper describes the design objectives, constraints, and implementation for the CESR accelerator upgrade for CHESS-U.
\end{abstract}

DOI: 10.1103/PhysRevAccelBeams.22.021602

\section{INTRODUCTION}

The Cornell Electron/positron Storage Ring (CESR) was designed as an electron-positron collider, with two diametrically opposed interaction regions. The layout of the storage ring is constrained by the very nearly circular geometry of the tunnel that it shares with the existing synchrotron. The CLEO detector required a long straight section to accommodate strong IR quads for a minibeta insert and antisolenoids to compensate the 1.5 T CLEO solenoid, in addition to the detector itself. Hard-bend dipoles were required to bend the trajectory into the long straight, with gentler bends immediately adjacent to the straight to mitigate synchrotron radiation background in CLEO. The collider layout and characteristic optics are shown in Fig. 1.

The Cornell High Energy Synchrotron Source (CHESS), founded contemporaneously with CESR, was initially comprised of three $\mathrm{x}$-ray extraction lines off the hard-bend dipoles from the counter-clockwise electron beam, expanding in 1988-89 with the addition of four beam lines (including one permanent-magnet wiggler) off the clockwise positron beam in CHESS East, and again in 1999 with the construction of G-line, adding three end stations fed by an insertion device from the positron beam.

Following the conclusion of the CLEOc HEP program in 2008, CESR was reconfigured for the CESR Test

*shanks@cornell.edu

Published by the American Physical Society under the terms of the Creative Commons Attribution 4.0 International license. Further distribution of this work must maintain attribution to the author(s) and the published article's title, journal citation, and DOI.
Accelerator (CesrTA) [1] damping ring R\&D program. The CLEO particle detector drift chamber was removed and the final focus replaced with a conventional FODO arrangement. The long straight was outfitted with six superconducting damping wigglers, relocated from two straights in the arcs.

The CesrTA layout also enabled the operation of CHESS in the arc-pretzel configuration with counter-rotating beams of electrons and positrons [2]. Layout and optics are shown in the middle plots in Fig. 1.

CHESS was designed to run parasitically with HEP operations, with beam lines fed by both electron and positron beams. Running with two electrostatically separated beams greatly complicates many facets of operation, including restricting bunch patterns and maximum perbunch current, increasing the tune plane footprint, and impairing the dynamic and momentum apertures.

With the conclusion of HEP operations, CESR is being reconfigured as a dedicated high-energy, high-performance single-beam synchrotron light source, CHESS-U. The conversion to operation with a single clockwise beam requires rebuilding the beam lines fed by the counterclockwise beam. The new layout eliminates the long IR straight and hard bends in favor of evenly distributed achromats and insertion straights, as shown in Fig. 1.

\section{LATTICE DESIGN}

\section{A. Design objectives and constraints}

The accelerator requirements for the CHESS-U upgrade are (1) Minimize the natural emittance for a single onaxis beam at $6.0 \mathrm{GeV}$; (2) Introduce straights for independent insertion devices for most end stations; (3) Preserve 

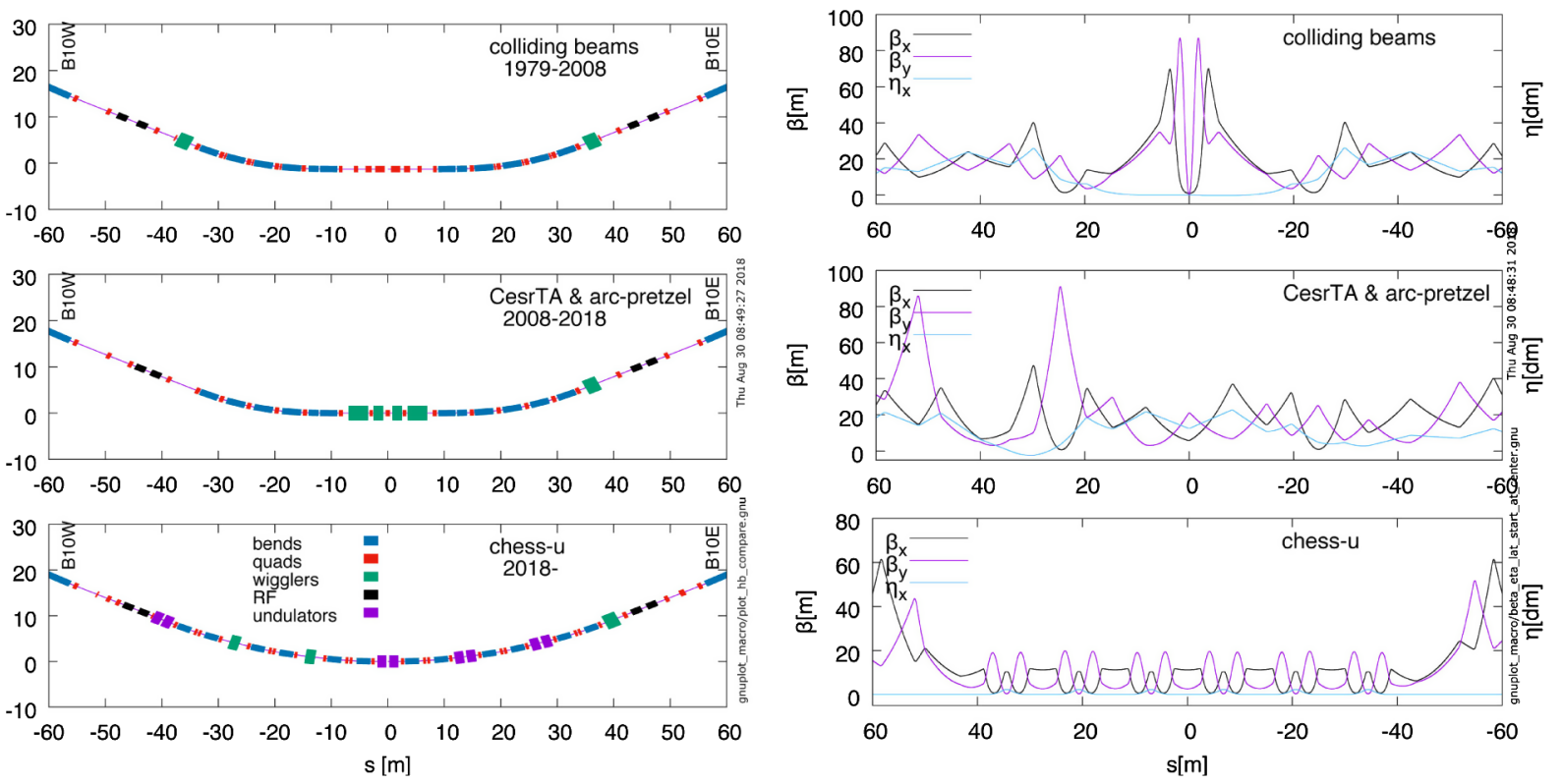

FIG. 1. At left is the magnetic layout of the L0 arc of the storage ring; (top) Colliding beams, (middle) CesrTA and CHESS arc-pretzel, (bottom) CHESS-U. At right optics in the L3 straight for colliding beams, arc-pretzel and CHESS-U.

compatibility with existing injector system (linac and synchrotron booster ring); and (4) Increase high-energy $\mathrm{x}$-ray flux for all end stations.

Most of the CHESS beam lines are presently located in the L0 main experimental hall, fed by both electron and positron beams, see Fig. 2. Eight end stations are located in L0, four from each beam; a further three end stations were added in 1999 as an excavation out of the west CESR tunnel, fed by the clockwise-oriented positron beam. For single-beam operation, a clockwise orientation was chosen in order to preserve the existing six end stations from the F-line and G-line facilities.

The existing storage ring layout in $\mathrm{L} 0$ includes one long straight section for the former CLEO detector, which is not optimal for low-emittance x-ray light source operation. The present optics do not have enough degrees of freedom to constrain the dispersion in the hard bend dipoles which bring CESR into the long straight, and as a result, the hard bends dominate the natural emittance (see Fig. 3). Additionally, the long straight section cannot accommodate multiple source points for x-ray beam lines, which require angular separation between sources.

The L0 experimental hall was also home to the three-story CLEO particle detector, which ran for HEP operation from 1979-2008. For single-beam CHESS operation, several $\mathrm{x}$-ray extraction lines would pass through the CLEO iron; to make room for new source points, the CLEO detector was disassembled and removed in 2016.

\section{B. Linear optics}

In order to maximize the number of end stations, a layout was chosen with six $13.8 \mathrm{~m}$ double-bend achromats with
$3.5 \mathrm{~m}$ insertion device (ID) straights. It is worth noting the cell length is approximately half that of many thirdgeneration storage ring light sources. DBAs were chosen over a multi-bend achromat design for a number of reasons: (1) The space available is extremely limited; (2) The dynamic aperture requirements would be incompatible with the existing accumulation injection scheme; and (3) the cost and complexity would be beyond the scope of this project.

The new DBAs utilize $2.35 \mathrm{~m}$ defocusing combinedfunction sector dipole-quadrupoles (DQs). Though the bend radius of the new CHESS-U dipoles is nearly identical to the old hard bends ( $31.4 \mathrm{~m}$ vs $31.7 \mathrm{~m}$ ), the dispersion is well suppressed in the new achromats, leading to a factor of four reduction in the global emittance while only replacing one-sixth of the storage ring.

A single DBA shown in Fig. 4. The natural emittance for a single CHESS-U cell is $2.56 \mathrm{~nm} \cdot \mathrm{rad}$ at $6.0 \mathrm{GeV}$. Matched into the remainder of the CESR, the full ring optics are shown in Fig. 5. Linear and nonlinear optics were optimized using Tao [3], which is built on the Bmad accelerator library [4].

The six new achromats span the 100-meter region between the two superconducting if straights, and begins and ends with straights for insertion devices. Five of the seven straights will be used for CHESS IDs; there is not presently space for end stations for the remaining two straights. The remaining two straights will have one CESRc superconducting damping wiggler each [5], to be used only in low-energy machine studies. The new CHESS-U lattice will enforce zero dispersion through the rf cavities, a condition which is not met in present two-beam CHESS optics. The new achromats are matched into the existing 


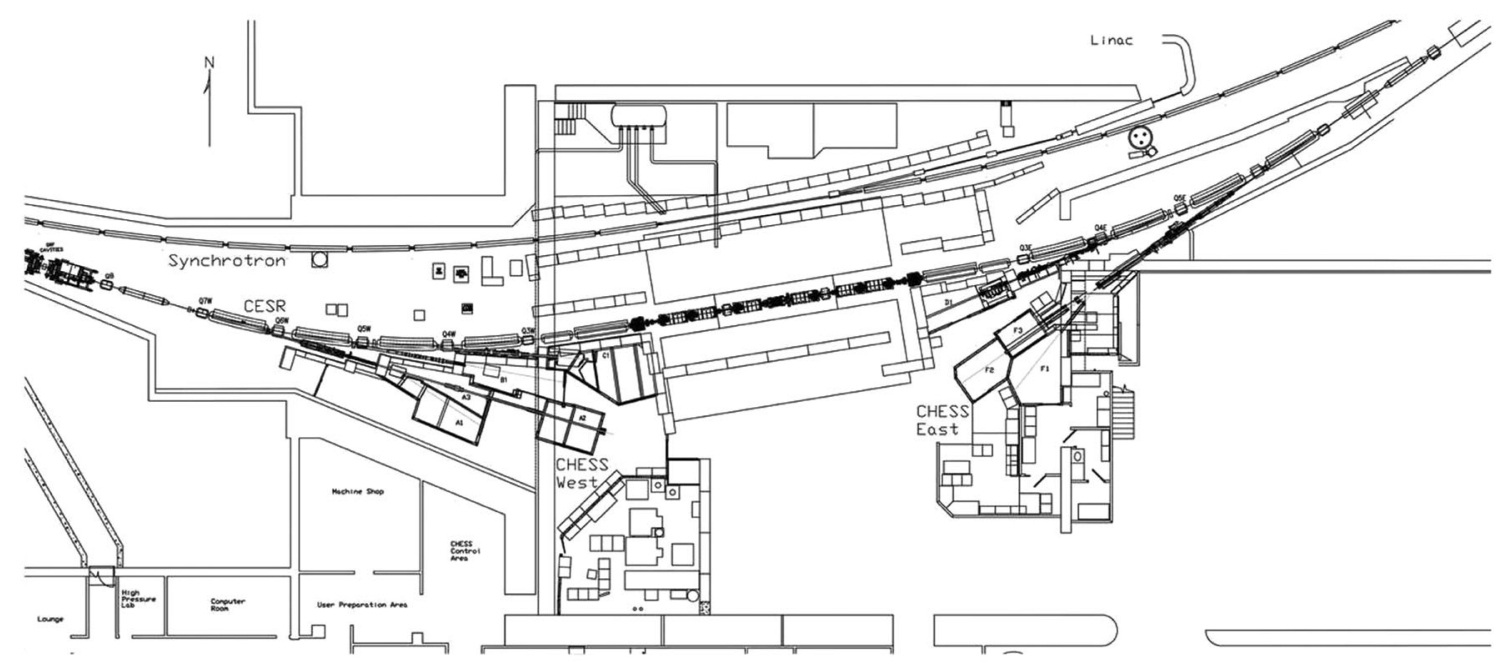

(a)

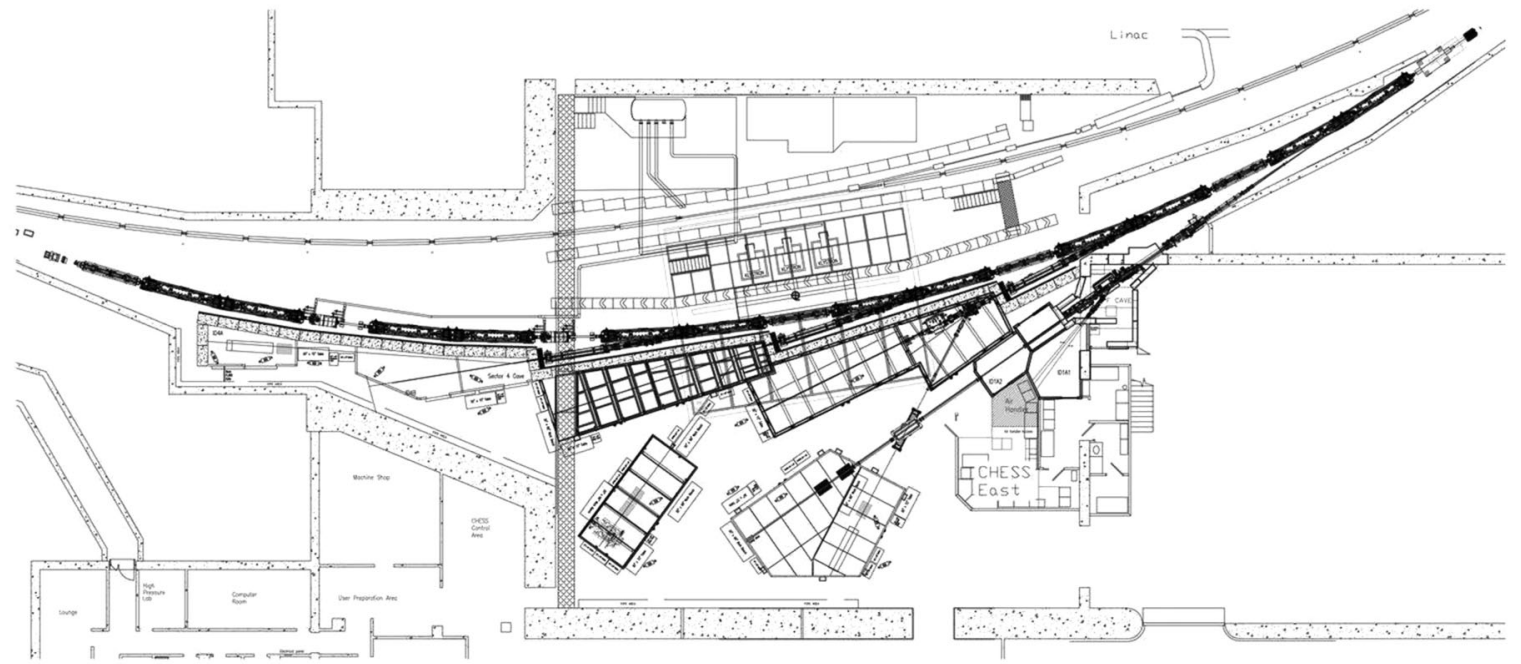

(b)

FIG. 2. CHESS layout, before and after CHESS-U upgrade. (a) Layout before CHESS-U; (b) CHESS-U layout.

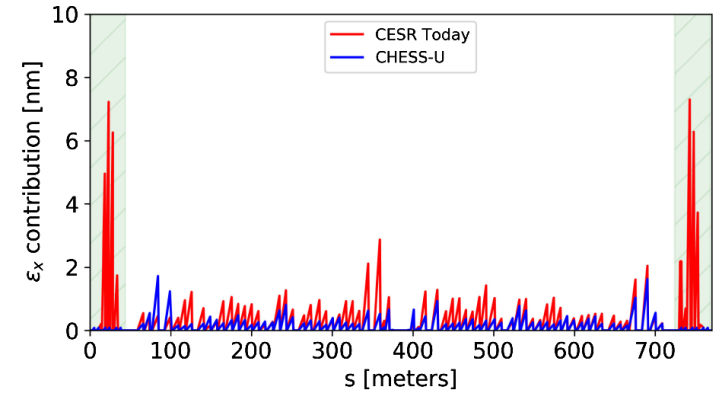

FIG. 3. Element-by-element contribution to the natural emittance, via the $I_{5}$ radiation integral. The green hatching indicates the region to be upgraded for CHESS-U. $s=0$ corresponds to the center of the former CLEO interaction region, which is also the center of the CHESS user region of the storage ring. The largest contributions to $I_{5}$ in the existing CESR layout correspond to the hard bend dipoles which bend into the long CLEO IR straight.

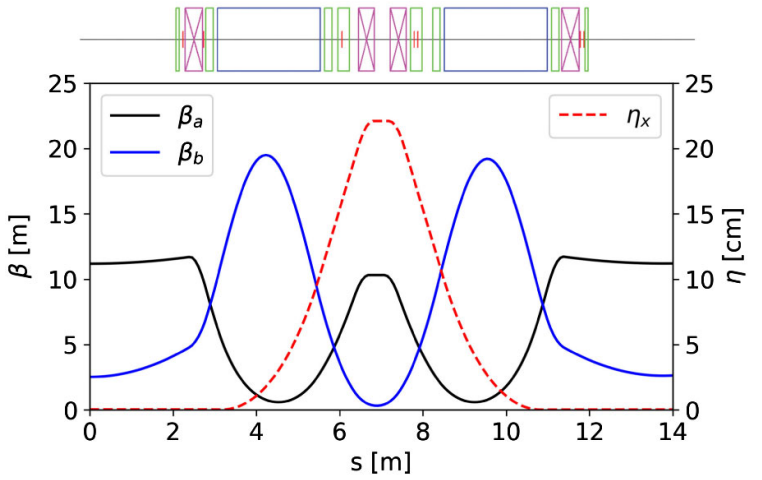

FIG. 4. One DBA cell for the CHESS-U upgrade. Blue boxes are DQs, purple are quadrupoles, green are steerings. Red ticks are beam position monitors. 


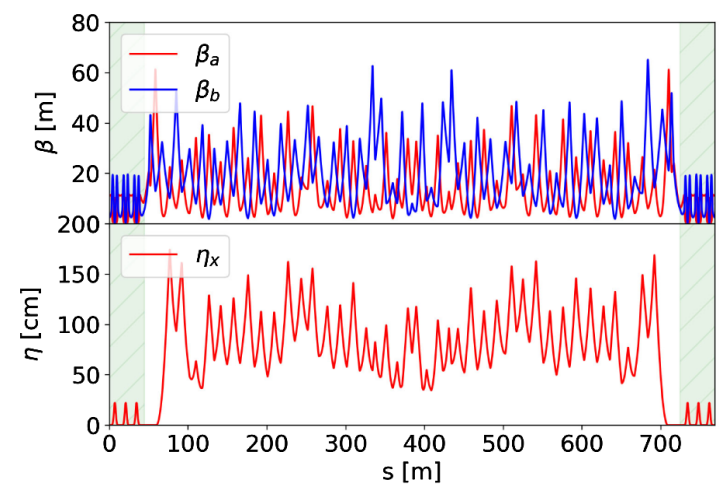

FIG. 5. Full ring optics for CHESS-U. $s=0$ corresponds to the center of the former CLEO interaction region, which becomes the center of the Sector 4 insertion device straight. The green hatching indicates the region being upgraded for CHESS-U.

FODO structure with four additional quadrupoles in each of the rf straights.

The storage ring energy will be increased to $6.0 \mathrm{GeV}$. Aside from CLEOc HEP operation in 2003-2008 and occasional CesrTA machine studies from 2008-2016, when the energy was lowered to $2.085 \mathrm{GeV} /$ beam, CESR has operated at $5.3 \mathrm{GeV} /$ beam. CHESS operation has been exclusively at $5.3 \mathrm{GeV}$. CESR was originally designed for an energy range of up to $8.0 \mathrm{GeV}$, though the highest operating energy to-date is $5.6 \mathrm{GeV}$. Machine studies conducted in 2017 in the present CESR configuration demonstrated injection and accumulation at $6.0 \mathrm{GeV}$. Every quadrupole and sextupole in CESR is independently powered, allowing significant flexibility in operating conditions. Therefore the remaining $5 / 6$ of the storage ring requires no modification.

CHESS-U lattice parameters are summarized in Table I.

TABLE I. Lattice parameters for CHESS today and CHESS-U upgrade.

\begin{tabular}{lccc}
\hline \hline Parameter & CHESS & CHESS-U & Units \\
\hline Circumference & 768.438 & 768.438 & $\mathrm{~m}$ \\
Energy & 5.289 & 6.0 & $\mathrm{GeV}$ \\
Species & $e^{+}$and $e^{-}$ & $e^{+}$ & \\
Current & $120 / 120$ & 200 & $\mathrm{~mA}$ \\
$\epsilon_{x}$ & 98 & 29.6 & $\mathrm{~nm} \cdot \mathrm{rad}$ \\
Emittance coupling & $1 \%$ & $1 \%$ & \\
$\beta_{x, y}$ at IDs & $7.9,3.1$ & $11.2,2.6$ & $\mathrm{~m}$ \\
$\eta_{x}$ at IDs & 0.42 & 0 & $\mathrm{~m}$ \\
IDs & 3 & 9 & \\
End stations & 11 & 12 & \\
$Q_{x, y}$ & $11.28,8.78$ & $16.55,12.63$ & \\
$Q_{x, y}^{\prime}$ & $9.2 \times 10^{-3}$ & $5.7 \times 10^{-3}$ & \\
$\alpha_{p}$ & 16 & 14 & $\mathrm{~mm}$ \\
Bunch length & 7 & 2.2 & $\mathrm{~mA}$ \\
Per-bunch current & $>24$ & 40 & $\mathrm{hrs}$ \\
Touschek lifetime & 5.2 & 6 & $\mathrm{MV}$ \\
RF Voltage & & & \\
\hline \hline
\end{tabular}

\section{Nonlinear optics}

Due to the lack of periodicity in CESR (the nearest symmetry in the ring is a mirror symmetry about the NorthSouth axis), nonlinear optics are numerically optimized for CHESS-U using 76 independently-controlled sextupoles with an implementation of J. Bengtsson's resonance driving term (RDT) formalism [6] in Bmad. As there is no symmetry, all sextupoles are allowed to vary separately.

An unusual design feature of the CHESS-U achromat is a lack of sextupoles in the new sextant. The peak dispersion in the DBAs is around $20 \mathrm{~cm}$, whereas the average dispersion in the remaining 5/6 of CESR is around $1 \mathrm{~m}$; the betas in the new achromats are also suppressed compared to the rest of the machine. As such, any sextupoles in the new sextant have little leverage on the chromaticity. Optimizations showed the inclusion of harmonic sextupoles in the new DBA cells did not significantly improve the nonlinear optics, and were therefore omitted.

The new DQ magnets have a significant $b_{2}$ multipole, contributing roughly $\mp 0.5$ to the horizontal and vertical chromaticity, respectively. This was compensated during the RDT reduction optimization.

\section{LATTICE CHARACTERIZATION}

\section{A. Performance}

Four of the five sectors with CHESS IDs will have canted 1.5 m CHESS Compact Undulators (CCUs) [7-9]. Sector 1 will use the existing 24-pole F-line wiggler [10]. Anticipated pinhole flux was simulated with SPECTRA [11], shown in Fig. 6.

\section{B. Error tolerance}

Error tolerance for CHESS-U was assessed using the tools and low-emittance tuning methodology developed for the CesrTA program [13]. The optics correction procedure used here is identical to that in CesrTA: (1) Measure closed

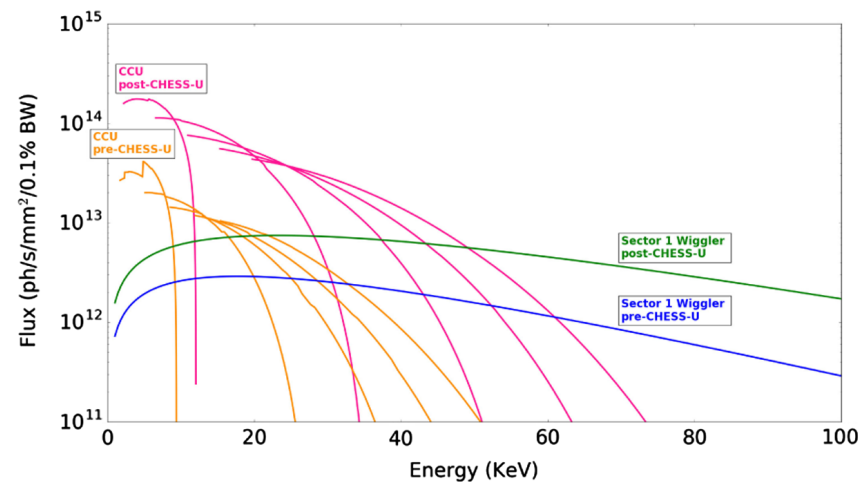

FIG. 6. SPECTRA modeling of flux through a $1 \mathrm{~mm}^{2}$ pinhole at 20 meters, before and after CHESS-U upgrade [12]. "F-line wiggler" refers to the 24-pole wiggler for Sector 1. 

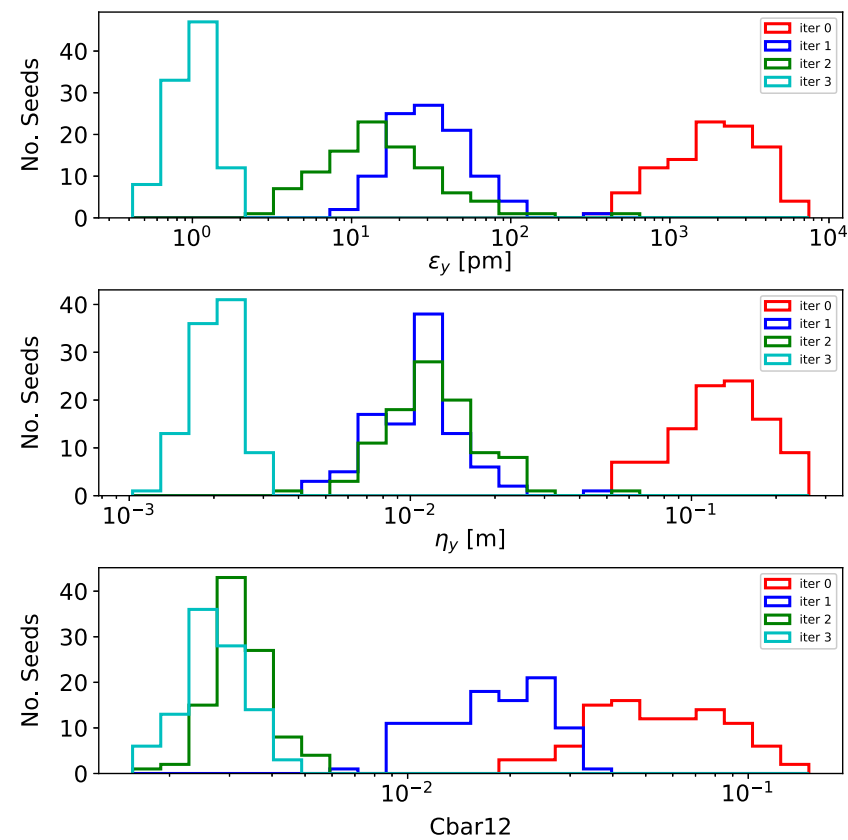

FIG. 7. Statistical analysis of 100 misaligned and corrected lattices. Iter 0 corresponds to the misaligned lattice before correction; the following three iterations correspond to the steps listed above. 95th-percentile $\epsilon_{y}=4.1 \mathrm{pm}$ after the final correction.

orbit; correct using horizontal and vertical steering magnets. (2) Measure betatron phase advance, local coupling [14], and horizontal dispersion; correct using quadrupoles and skew quadrupoles. (3) Measure orbit, local coupling, and vertical dispersion; correct using vertical steerings and skew quadrupoles.

Though the new DQs have trim windings, the correction simulation did not allow for the DQs to vary. Error amplitudes are listed in Tables V and VI, and represent the best estimate of anticipated alignment and BPM errors.

A summary of the misalignment study is shown in Fig. 7. The statistical impact of misalignments and corrections on the Twiss parameters is shown in Fig. 8. The 95thpercentile vertical emittance due to optics errors after correction is $4.1 \mathrm{pm}$.

\section{Dynamic and momentum aperture}

Dynamic and momentum aperture were evaluated via frequency map analysis [15]. Particles were tracked for a total of 2048 turns at each initial amplitude; dynamic aperture plots were normalized to beam sigmas, assuming $1 \%$ emittance coupling.

Systematic multipoles for dipoles, quadrupoles, and sextupoles were determined via field modeling or measurement, and are listed in Table VII. Due to the compact nature of the CCU magnet arrays, the field roll-off is significant: vertical field is reduced $10 \%$ at a $10 \mathrm{~mm}$ horizontal displacement. Systematic multipoles and the
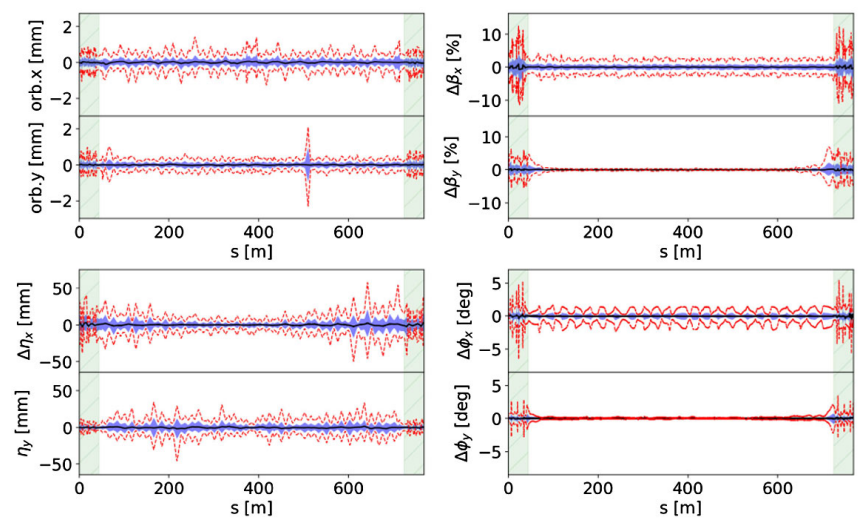

FIG. 8. Statistics of machine optics distortion as a function of longitudinal position for 95 out of 100 misaligned and corrected lattices (representing 95th-percentile anticipated results). Black curve indicates the average over 100 seeds, blue shading indicates the standard deviation, and the red dashed lines indicate the maximum and minimum excursion at any given point in the lattice. The green hatched region indicates the region with new CHESS-U achromats, where betas and dispersion are suppressed compared to the rest of the ring.

field roll-off and measured field integrals for CCUs were included in some tracking simulations [2], denoted in the relevant figure captions.

Dynamic aperture and its footprint in the tune plane are shown in Fig. 9 for combinations of systematic multipoles, insertion devices, and misalignment/correction; corresponding momentum aperture with systematic multipoles, IDs, and misalignment/correction are shown in Fig. 10. The color scale in these plots is the tune diffusion, given by

$$
d Q=\log \sqrt{\Delta Q_{x}^{2}+\Delta Q_{y}^{2}}
$$

\section{Injection simulation}

The injection scheme for CESR relies on accumulation from a $60 \mathrm{~Hz}$ synchrotron booster ring. Injection efficiency was simulated via multiparticle tracking. 200 macroparticles were launched with a phase space matching the beam at the end of the synchrotron booster extraction line. Particles which survive for 400 turns were considered captured. Details are found in [2].

Results of the injection tracking simulations are shown in Fig. 11. It should be noted that the injection efficiency is not expected to reach $100 \%$ as there are two $10 \mathrm{~mm}$ fullaperture vertical collimators used to mask against particle losses on the small-gap permanent-magnet IDs.

\section{MAGNET DESIGN}

Most constraints on magnet design are more or less standard. The specific design impacts on the CHESS-U magnets are discussed here. Details are available in [16]. 

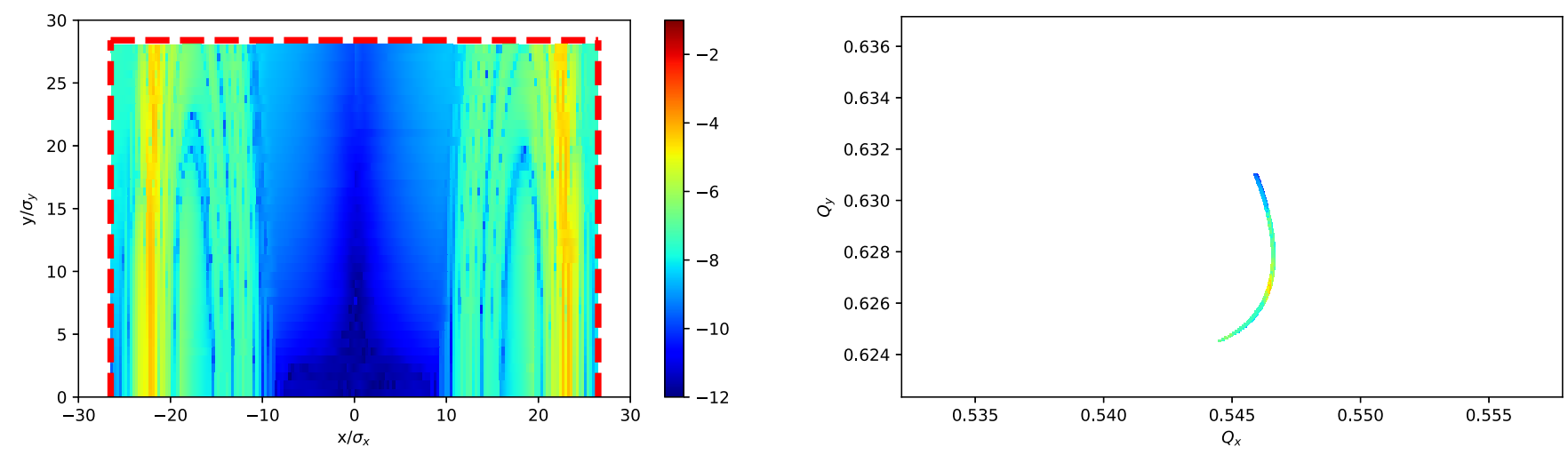

(a)
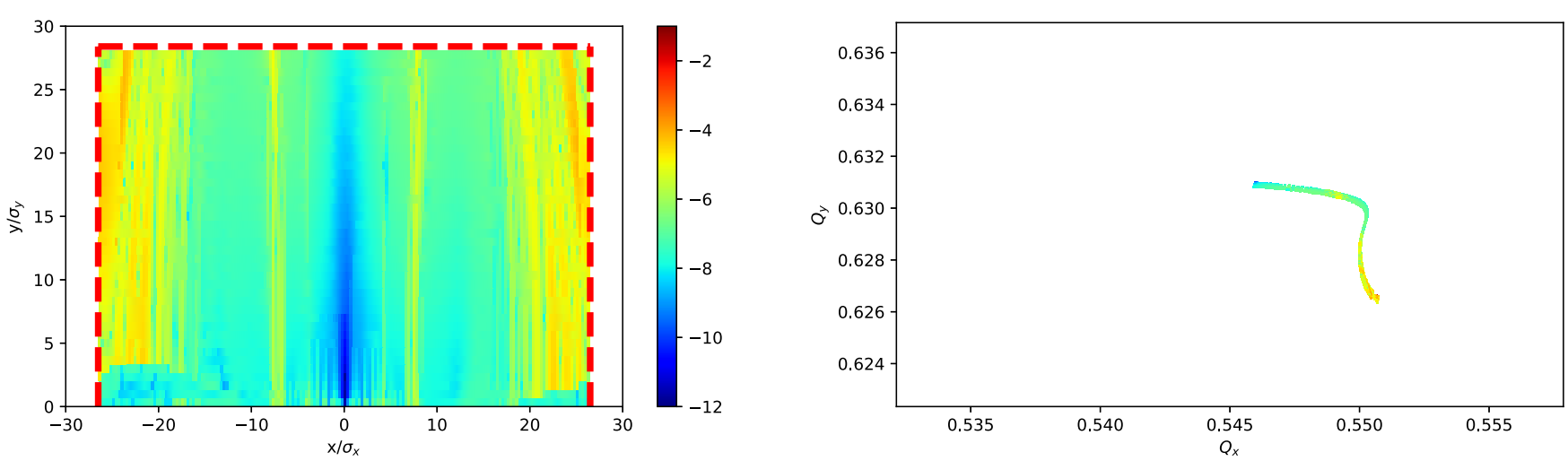

(b)
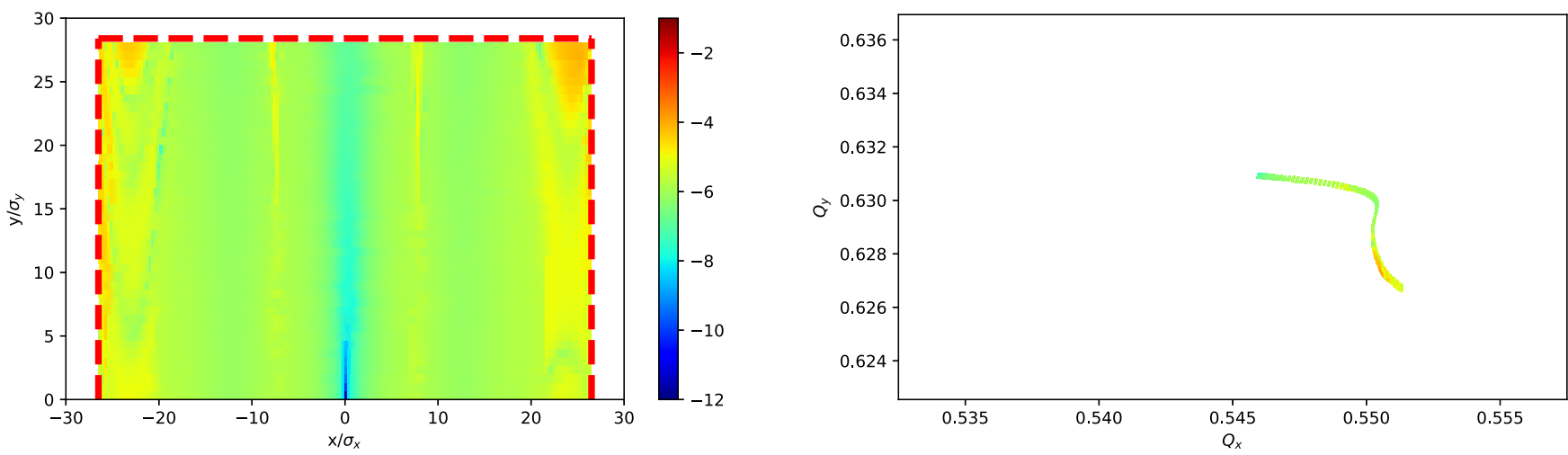

(c)

FIG. 9. Dynamic aperture via the frequency map (left column), and its associated footprint in the tune plane (right column). Color scale indicates tune diffusion, defined in Eq. (1). Red dashed line indicates the minimum projected aperture. (a) Ideal lattice; (b) With systematic multipoles and realistic undulator field tracking; (c) With systematic multipoles, realistic undulator field tracking, and misalignments and corrections (95th-percentile seed).

\section{A. Combined function dipole-quadrupoles}

Space constraints and emittance reduction motivated implementing DQs in the new DBA cells rather than conventional dipoles. The DQs have a magnetic length of $2.35 \mathrm{~m}$, bend radius of $31.4 \mathrm{~m}$, and gradient $-8.77 \mathrm{~T} / \mathrm{m}$. The full-gap aperture along the design trajectory is $38.4 \mathrm{~mm}$. The 3D OPERA model and excitation curve for the DQ magnet are shown in Fig. 12. Although the magnet yoke is rectangular in geometry, the pole pieces follow a sector dipole trajectory.

\section{B. Quadrupoles}

All achromat quadrupoles are horizontally-focusing, as the vertical focusing is done by the DQs. There are two families of 

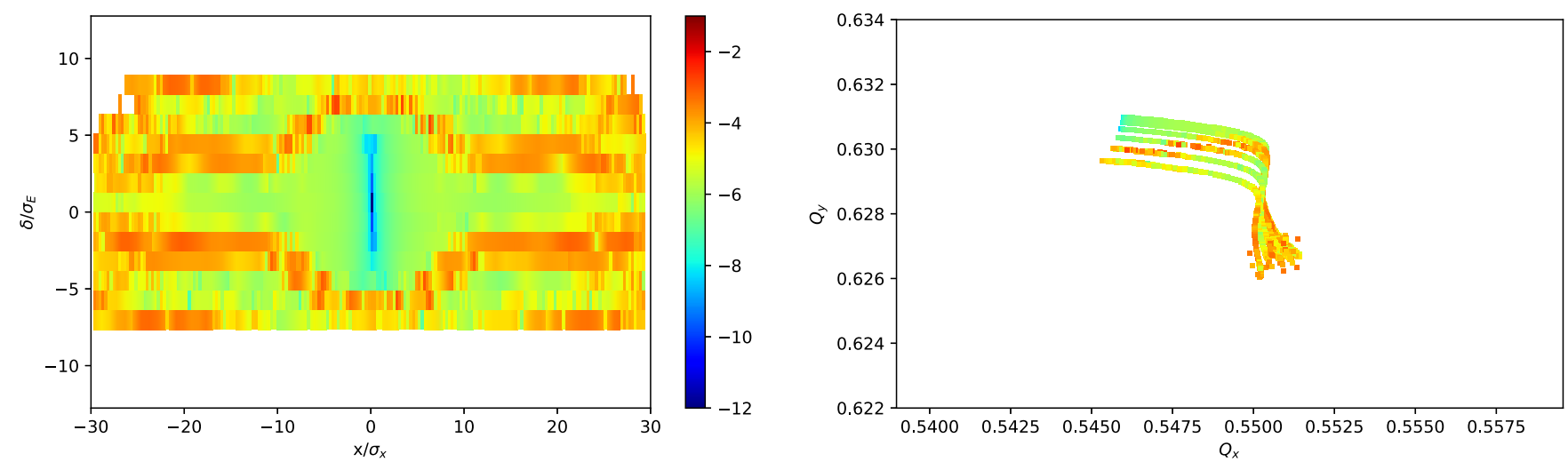

FIG. 10. Momentum aperture via the frequency map and its associated footprint in the tune plane, with systematic multipoles, realistic undulator field tracking, and misalignments and corrections (95th-percentile seed). Color scale indicates tune diffusion, defined in Eq. (1).

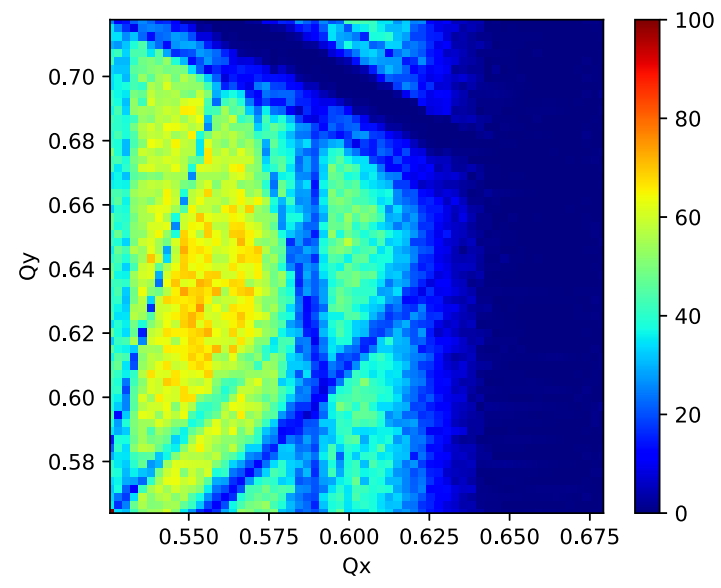

FIG. 11. Injection tune scan simulation with systematic multipoles, realistic undulator field tracking, and misalignments and corrections (95th-percentile seed). Color scale indicates capture efficiency in percent.

quadrupoles, with magnetic lengths $0.400 \mathrm{~m}$ and $0.362 \mathrm{~m}$, both with the same pole profile; the primary distinctions are length and the extension of the iron to allow for x-ray extraction on one of the two families. Both quadrupole families have maximum gradient $39 \mathrm{~T} / \mathrm{m}$, with a bore radius of $23 \mathrm{~mm}$. Figure 13 shows the magnetic field in one quarter of a nonextraction quadrupole and the excitation curve; an extraction quad model is shown in Fig. 14.

\section{CHESS compact undulators}

Four of the five new sectors will be equipped with canted pairs of CHESS compact undulators (CCUs). The design for the CCUs is detailed elsewhere [7-9]. An end-view of the CCU profile is shown in Fig. 15(a); perspective CAD drawing of CCU assembly is shown in Fig. 15(b). Parameters for the CCUs are summarized in Table II.
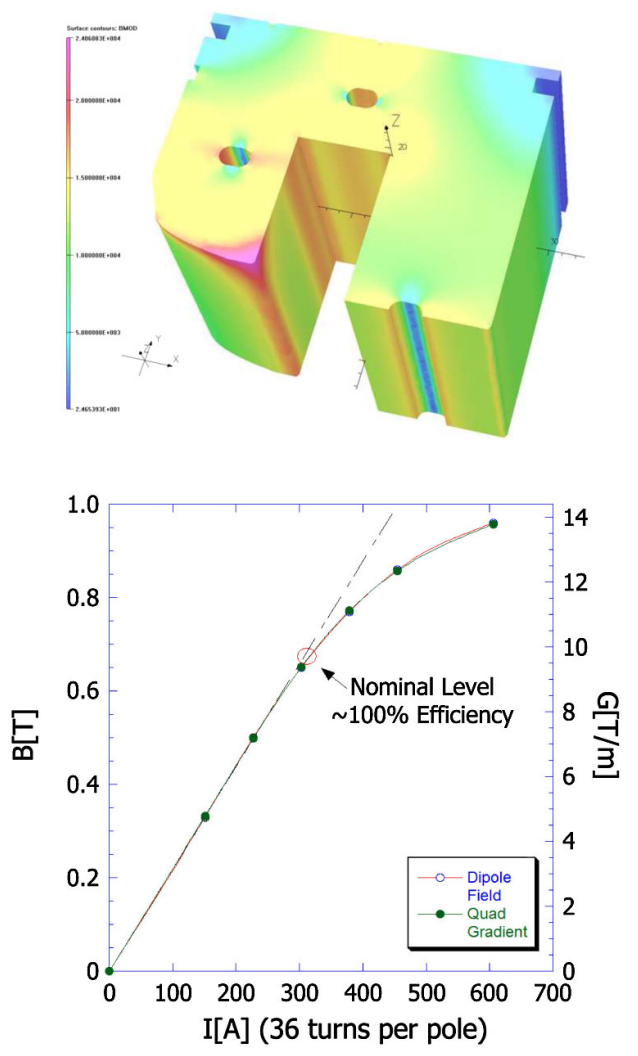

FIG. 12. Top: Magnetic field in one half of a DQ iron. Peak field in the iron is approximately $2.3 \mathrm{~T}$. Bottom: DQ excitation curve.

The CCUs utilize a variable-phase design rather than variable-gap in order to minimize the supporting infrastructure. As implemented in CESR the CCUs have a $7.0 \mathrm{~mm}$ out-of-vacuum vertical aperture between poles, mandating the vacuum chamber to fit inside this aperture. The associated vacuum chamber for the CCUs is described in Sec. V B. 

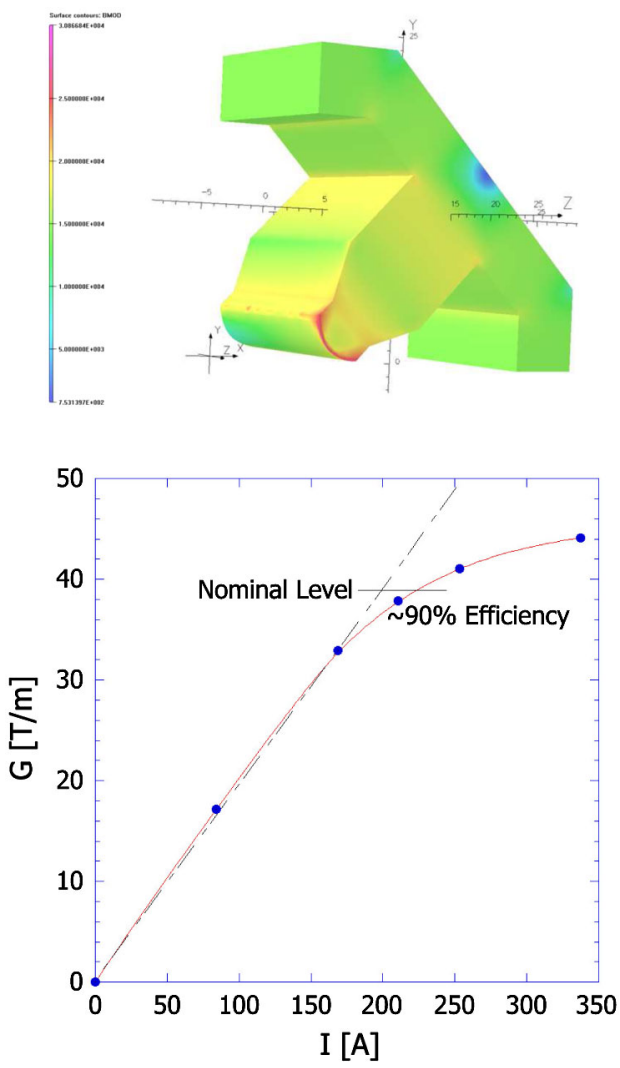

FIG. 13. Top: Magnetic field in one quarter of a nonextraction quadrupole iron. Peak field in the iron is approximately $2.3 \mathrm{~T}$. Bottom: Quadrupole excitation curve.

CHESS has operated with two $1.5 \mathrm{~m}$ CCUs in a canted straight since 2014, with two counterrotating beams. Total beam current has routinely exceeded the CHESS-U target $200 \mathrm{~mA}$ in single-beam positron tests at the present CHESS beam energy of $5.3 \mathrm{GeV}$.

\section{Power distribution}

For the new CESR south arc, the magnet power distribution was chosen to be compatible with existing

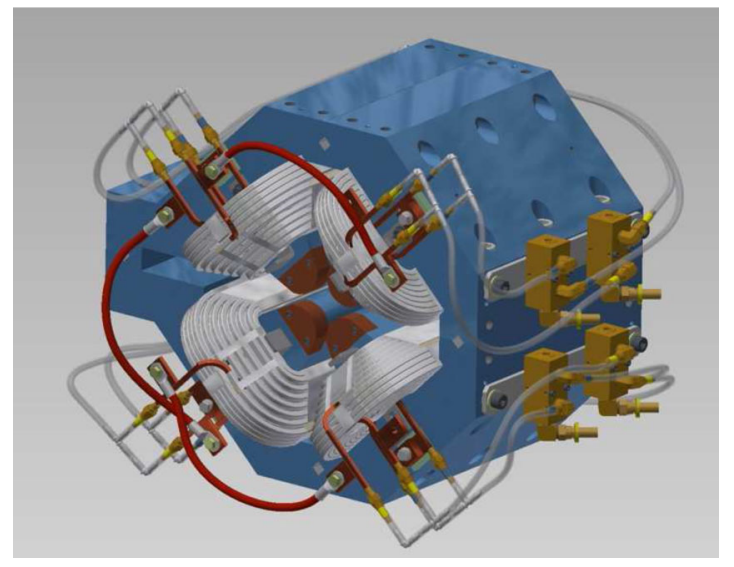

FIG. 14. Extraction quadrupole model.

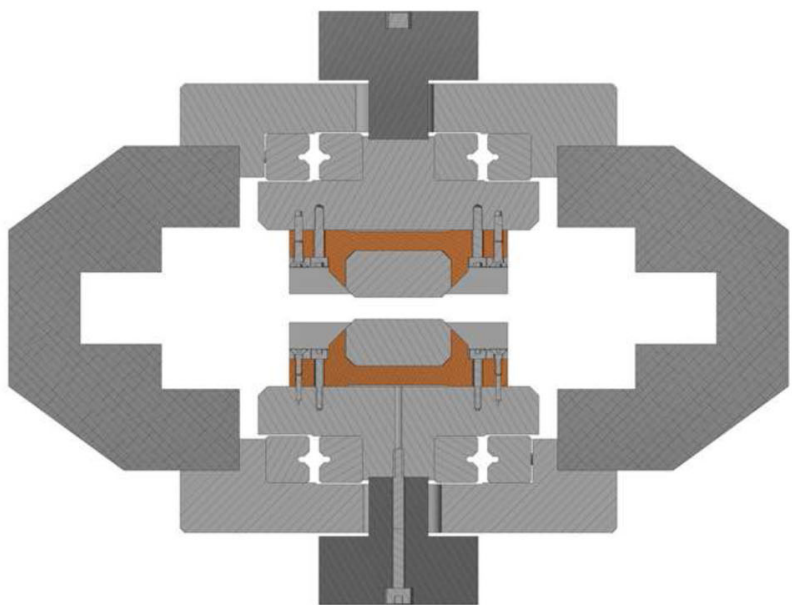

(a)

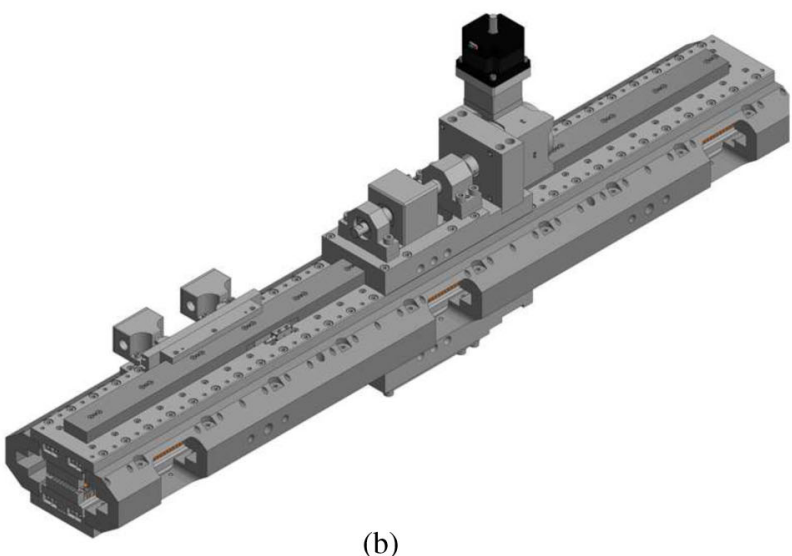

FIG. 15. CAD model of the Cornell Compact Undulator (CCU). (a) End-on view; (b) Perspective view.

infrastructure. There are two main pieces to this: the CESR dipole circuit and the CESR quadrupole bus system. The controls for both are derived from a unique computer bus known as the Xbus [17,18]. The Xbus handles all digital and analog controls, read back signals, and interlocks associated with the operation of the magnet power supplies. All magnets can be controlled individually or collectively as needed from the central control room.

The CESR Dipole circuit is a series circuit of approximately 120 bend magnets driven by two $0.5 \mathrm{MW}$ Transrex power supplies at $500 \mathrm{~V}$ and $740 \mathrm{~A}$ to obtain $6 \mathrm{GeV}$. The series chain is made up of normal bend, soft bend, and

TABLE II. Parameters for the CHESS Compact Undulators.

\begin{tabular}{lc}
\hline \hline Parameter & Value \\
\hline Length & $1.477 \mathrm{~m}$ \\
Period & $28.4 \mathrm{~mm}$ \\
Peak field & $0.952 \mathrm{~T}$ \\
Pole gap & $7.0 \mathrm{~mm}$ \\
First harmonic & $2.9 \mathrm{keV}$ \\
\hline \hline
\end{tabular}




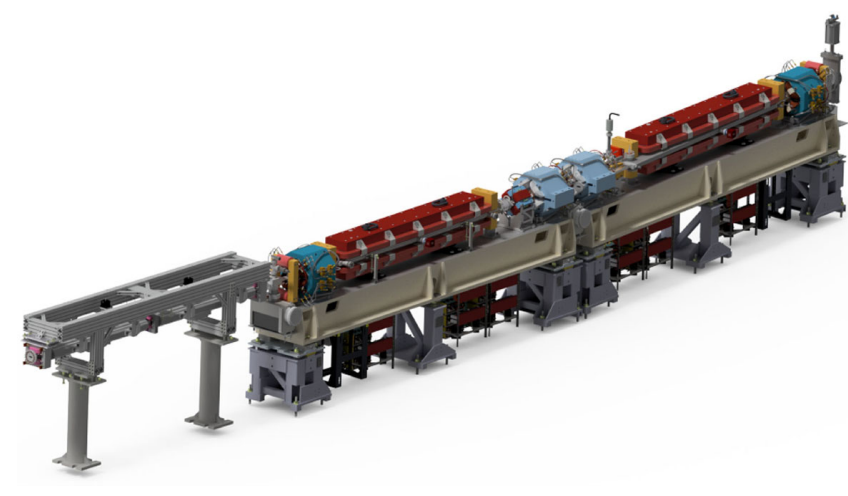

FIG. 16. Rendering of one sector for CHESS-U from the radialoutside. Beam travels clockwise (right-to-left).

hard bend dipole magnets. The new DQ magnets will be part of this existing circuit.

The operation of CHESS-U requires five quadrupole buses: East, South East, South Center, South West, and West. The SE, SC, and SW are new buses installed expressly for the south arc installation. The West and East quad buses are preexisting. Each quad bus is driven by an $80 \mathrm{~V} 1125$ A EMHP power supply. These buses supply a fixed $70 \mathrm{~V}$ primary voltage to a large number of switching power supplies (DC-to-DC converters). These power supplies excite all quadrupole, sextupole, steering, skew quadrupole, skew sextupole, and octupole magnets. Through the Xbus, each switching power supply can be controlled independently, giving flexibility for tuning optics. The current draw depends on the optics, typically scaling with beam energy.

\section{E. Support and alignment}

Each new CHESS-U cell was split into three sections: an upstream girder, a downstream girder, and an insertion device straight. The two girders support all achromat magnets, each holding two quadrupoles and one DQ, plus corrector magnets. The insertion device straight is suspended from above in order to allow access to the upstream sector's front end. One sector is illustrated in Fig. 16.

Magnetic alignment of the DQ and quadrupoles is achieved using a combination of vibrating wire [19] and Hall probe. The procedure is as follows: (1) Establish vibrating wire $(\mathrm{VW})$ position with respect to girder fiducials (2) Establish Hall probe position with respect to VW

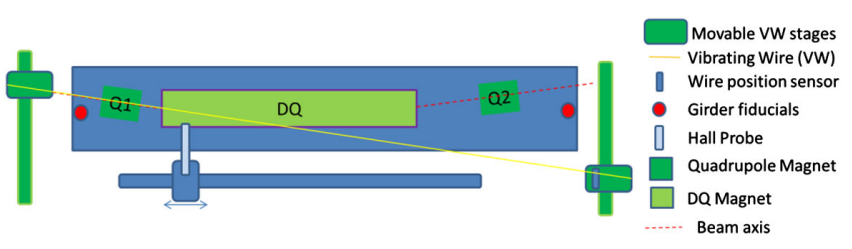

FIG. 17. Setup for magnetic centering of DQ and quadrupoles on a girder. [16].

(3) Place wire on beam axis (4) Align Q1 magnetic axis with VW (5) Align Q2 magnetic axis with VW (6) Align DQ using Hall probe.

The setup used for this alignment procedure is diagrammed in Fig. 17. Using this method, the magnetic centers of quadrupoles and the DQ on one girder are aligned to within $32 \mu \mathrm{m}$.

\section{VACUUM DESIGN}

\section{A. Design considerations}

As a part of particle beam transport system, the CHESS-U vacuum chambers need to provide adequate physical beam aperture, while maintaining sufficient clearance to all CHESS-U magnets. The vacuum pumping system is designed to achieve and to maintain ultrahigh vacuum (UHV) conditions, with an average vacuum pressure $<1$ nTorr with stored $200 \mathrm{~mA}$ beam at $6.0 \mathrm{GeV}$. Additionally, all vacuum chambers must be designed to manage heating from synchrotron radiation (SR) generated by the bending magnets, with a minimum factor of safety $(\mathrm{FOS})>2$. The vacuum chambers will also house a suite of BPMs for beam instrumentation.

\section{B. Vacuum chambers}

All CHESS-U vacuum chambers are constructed from type 6061 and 6063 aluminum alloys. Similar to the modular magnet design concept, the CHESS-U vacuum chambers conform to the periodic achromat cells. Owing to the limited longitudinal spaces between the magnets, the vacuum string for each achromat is made into three flanged chambers, as shown in Fig. 18. More specifically, each achromat is comprised of two long dipole chambers, a $4.91 \mathrm{~m}$ long downstream "Dipole C" chamber and a $5.03 \mathrm{~m}$ long upstream "Dipole A" chamber, which are integrated with magnet girders, and a $3.69 \mathrm{~m}$ long undulator vacuum chamber.

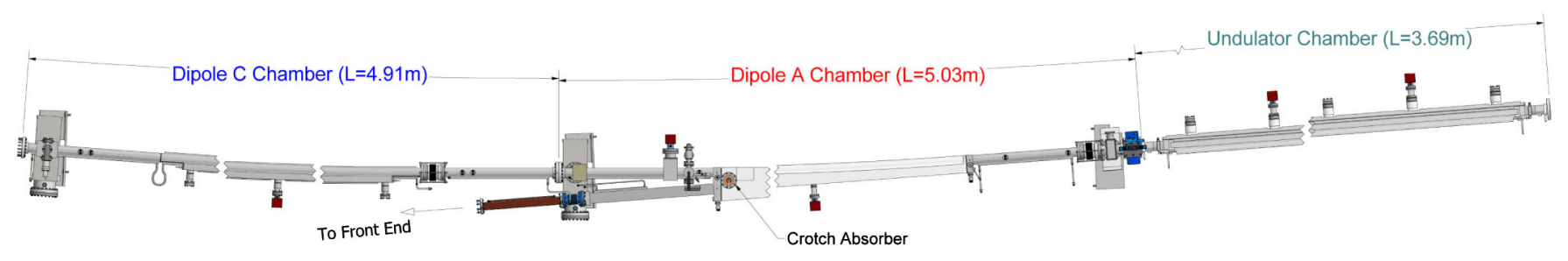

FIG. 18. A typical CHESS-U Achromat Cell vacuum chamber string is consisted of three long vacuum chambers. 

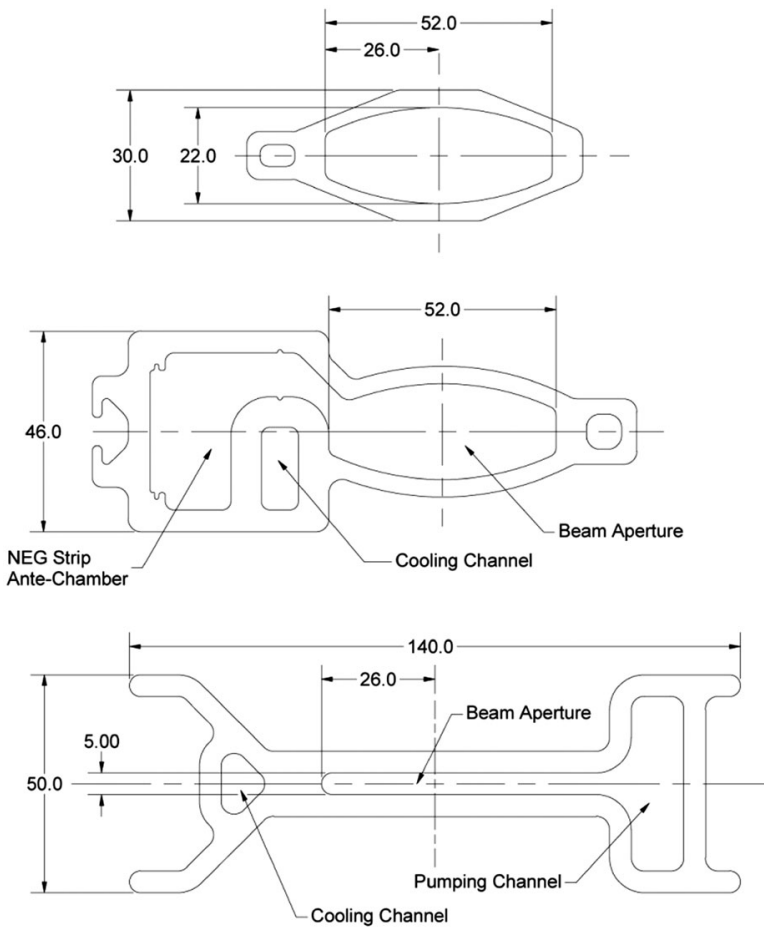

(a)
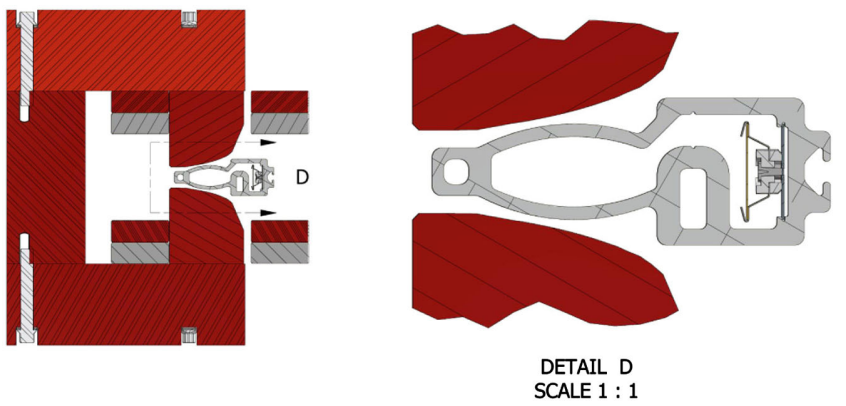

(a)
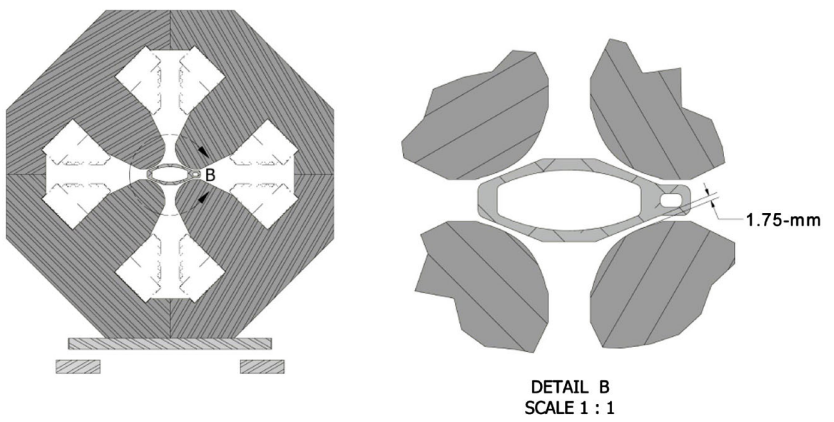

(b)

FIG. 20. Cross sections of extrusions in their respective magnets. (a) Dipole extrusion in a DQ magnet; (b) Quadrupole extrusion in a quadrupole magnet.

The dipole A chamber design is much more complicated than the dipole $\mathrm{C}$ chamber, as it needs to provide egress for the X-ray from the canted undulators. The dipole A design is illustrated in Fig. 21(b). To incorporate the compact geometry needed for the passage of both stored electron beam and egress of X-ray from the undulators, the chamber main body is constructed by welding of two precision machined "clam-shells" of 6061-T6 aluminum alloy. Cooling channels and NEG strip mounting features are also machined in the main body. Similar to the dipole C chamber, quad extrusions are welded to both ends of the main body. Two sets of BPMs, one rf-shielded sliding joint and multiple pump ports are also directly welded to the chamber. At separation junction of the electron beam and the x-ray, an insertable crotch absorber is mounted onto the dipole A chamber, to intercept up to $5.4 \mathrm{~kW}$ of SR power from the dipole magnet, while allowing required passages of both electron beam and the X-ray. The crotch absorber is one of the most challenging CHESS-U vacuum components, due to very limited available space. Its design is based on the existing CHESS crotches, consisting of a pure beryllium ring ( $5 \mathrm{~mm}$ thick) and a water-cooled copper core. The beryllium ring, vacuum brazed to the copper core, dilutes the SR power density by bulk absorption and scattering along its depth. FEA analysis indicates that the crotch absorber has sufficient margin of safety to handle the SR power from the design CHESS-U beam parameters $(6.0 \mathrm{GeV}, 250 \mathrm{~mA})$. Design and construction of 


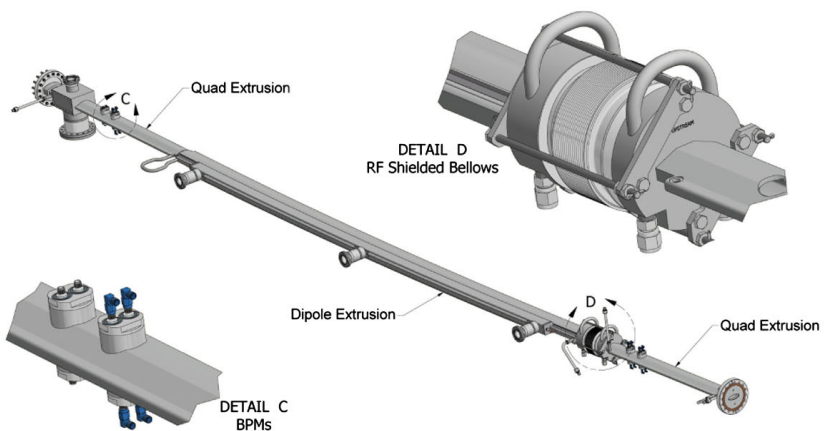

(a)

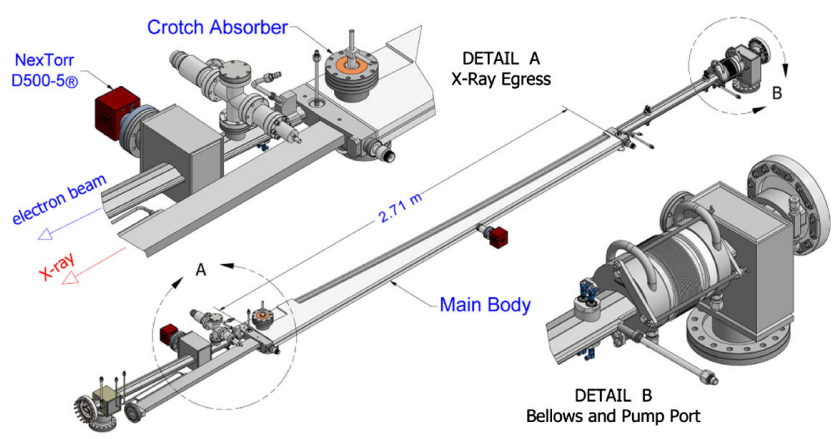

(b)

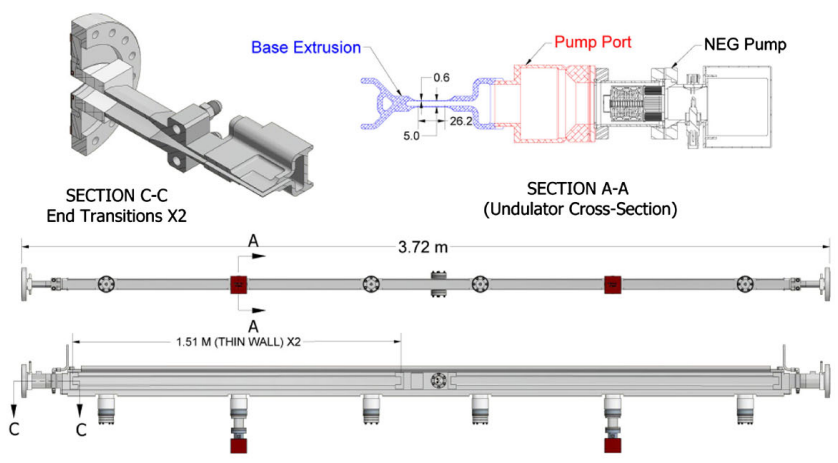

(c)

FIG. 21. Vacuum chamber detail. (a) Dipole $\mathrm{C}$ vacuum chamber; (b) Dipole A vacuum chamber; (c) Undulator vacuum chamber.

the CHESS-U undulator chambers are similar to an existing $\mathrm{CCU}$ chamber in operation for the last four years. As shown in Fig. 21(c), the undulator beampipe is made from the CHESS-U undulator extrusion [Fig. 19(c)]. Two sections (1.51 m each) of the extrusion are machined to a thickness of $0.61 \mathrm{~mm}$ to accommodate the $7.0 \mathrm{~mm}$ CCU pole gap. The machining of the undulator extrusion is done dry to avoid difficulty in cleaning the long beampipe with $5 \mathrm{~mm}$ vertical aperture with thin walls. The wall thickness at the thin section was constantly checked using ultrasonic thickness gauges during the machining. Two end assemblies are welded to the undulator beampipe, that gently transition vertically from $5 \mathrm{~mm}$ to $22 \mathrm{~mm}$, the nominal CHESS-U

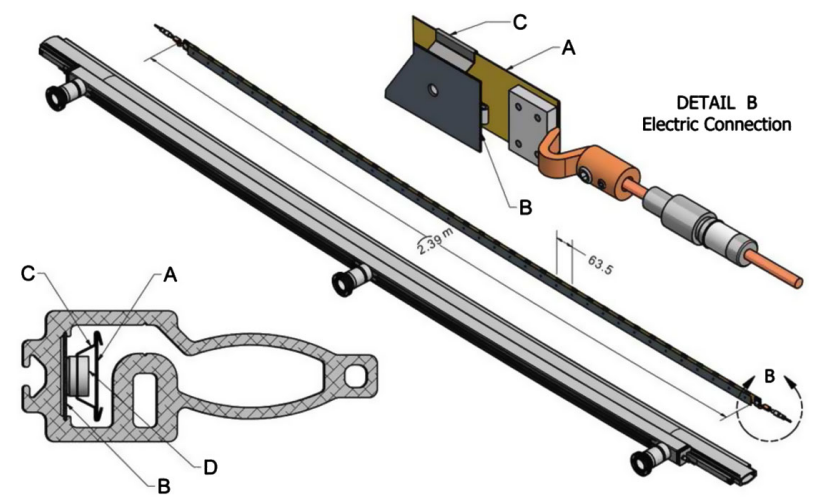

FIG. 22. NEG strip assembly in dipole chambers. (A) 30-mm NEG (SAES Getters st-707) strip; (B) Stainless steel carrier ribbon; (C) Stainless steel clips; (D) Alumina spacers.

vertical aperture. Six pumping ports are welded to the antechamber of the undulator extrusion.

\section{Vacuum pumping and performance simulation}

All CHESS-U vacuum chambers were baked to $150{ }^{\circ} \mathrm{C}$ and then back-filled with ultrahigh purity nitrogen through a MATHESON NANOCHEM ${ }^{\circledR}$ purifier that reduces $\mathrm{H} 2 \mathrm{O} / \mathrm{HC}$ level below ppb level. A post-installation $95^{\circ} \mathrm{C}$ hot water bakeout of all CHESS-U chambers is also planned. With these measures, SR-induced desorption (SRID) is expected to be the dominant source of gas load.

Owing to distributed nature of the SRID gas load and restricted conductance of the CHESS-U beampipe, it is essential to have distributed vacuum pumping. Nonevaporable getter (NEG) strips (st-707 SAES Getters) were chosen for the distributed pumps in the dipole chambers over distributed ion pump in CESR, due to the limited space available. The structure of the NEG strip assembly is shown in Fig. 22. A $30 \mathrm{~mm}$ wide NEG strip is supported on a stainless steel ribbon with periodic stainless steel clips. The clips are pinched to the NEG strip, and are riveted to the stainless steel ribbon through sets of alumina spacers. The NEG strip will be activated by resistive heating to $500{ }^{\circ} \mathrm{C}$ with a DC power supply. Flexible connections at both ends of the NEG strip to the electric vacuum feedthroughs allow thermal expansion during activation.

Very high SRID gas load is expected in the initial beam conditioning stage. Simulations showed that the NEG strips may not have sufficient pumping capacity during the CHESS-U commissioning phase, and it can be disruptive to the initial operation with frequent activation cycles. Therefore, compact high capacity NEG modular pumps, CapaciTorr Z200 and NexTorr Z100 (SAES Getters), are installed to aid the initial beam conditioning. To handle potential noble (or non-gettable) gases, a large sputtering ion pump (approx. $110 \mathrm{l} / \mathrm{s}$ ) is also installed on each achromat cell. 


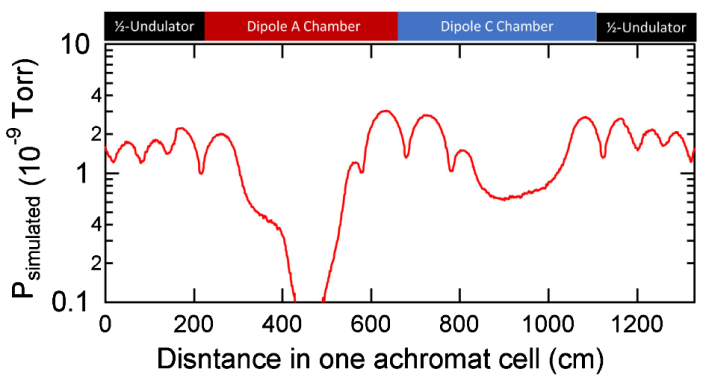

FIG. 23. Simulated CHESS-U pressure profiles with $250 \mathrm{~mA}$ stored beam with $100 \mathrm{~A}-\mathrm{hr}$ of beam-conditioning, assuming NEG pumps at nominal pumping speed, using MolFlow+, which showed an average pressure of $\approx 1.8 \times 10^{-9}$ torr.

The CHESS-U vacuum pumping performance was evaluated using MolFlow+, a Test-Particle Monte-Carlo simulator developed at CERN. The simulations showed that the installed vacuum pumping system is capable of achieving required level of vacuum for CHESS-U operations. Figure 23 displays an example of simulated pressure profile in one CHESS-U achromat cell, after $100 \mathrm{Amp}-\mathrm{hr}$ beam conditioning, achieving an average pressure of $\approx 1.8 \times 10^{-9}$ torr. Pressure bumps are due to lack of pumping in the straight sections populated by the magnets.

\section{INSTRUMENTATION}

CESR is well-instrumented for a multitude of beambased measurement techniques, thanks to the CesrTA program. Details are documented elsewhere [20]. A few highlights are noted here.

\section{A. CESR beam position monitors}

CESR is presently equipped with 110 peak-detection BPMs of an in-house design (CBPM-II), capable of measuring bunch-by-bunch turn-by-turn positions for bunch spacings $\geq 4 \mathrm{~ns}$. $100 \mathrm{CBPMs}$ are used for routine optics correction and orbit monitoring. Each achromat in CHESS-U will be instrumented with four CBPM-II modules, one adjacent to every quadrupole. CBPM-II specifications are listed in Table III; further details are available in [21].

With the installation of canted CCU undulators in 2014 [22], three Libera Brilliance Plus processors have been

TABLE III. CBPM-II specifications.

\begin{tabular}{lc}
\hline \hline Parameter & Specification \\
\hline Front-end bandwidth & $500 \mathrm{MHz}$ \\
Absolute position accuracy (long-term) & $100 \mu \mathrm{m}$ \\
Single-shot position resolution & $10 \mu \mathrm{m}$ \\
Differential position accuracy & $10 \mu \mathrm{m}$ \\
Channel-to-channel sampling time resolution & $10 \mathrm{psec}$ \\
\hline \hline
\end{tabular}

used for continuous turn-averaged position monitoring at the existing $3.5 \mathrm{~m}$-long $4.6 \mathrm{~mm}$-aperture $\mathrm{CCU}$ chamber.

\section{B. X-ray video beam position monitors (vBPMs)}

Each x-ray front end will be equipped with two vBPMs, one for each extracted beam, to provide horizontal and vertical position. The vBPMs will use an edge-on diamond blade monitor which was prototyped on A-line in 2015 [23], rendered in Fig. 24. The diamond-blade style vBPM allows for a compact design close to the source (roughly $10 \mathrm{~m}$ ), where the canted undulator beams are only separated by $10 \mathrm{~mm}$.

The vBPMs are presently used in conjunction with Libera BPMs around the small-gap undulator chamber to monitor x-ray trajectories in real-time and correct positions after every topoff, typically every five minutes. This strategy will be expanded in CHESS-U, where each sector will have two Libera Brilliance Plus monitors dedicated to real-time position monitoring for position feedback.

\section{Turn-by-turn feedback}

Bunch-by-bunch turn-by-turn horizontal, vertical, and longitudinal feedback is accomplished using three Dimtel feedback front-end and processing modules [24]. The low

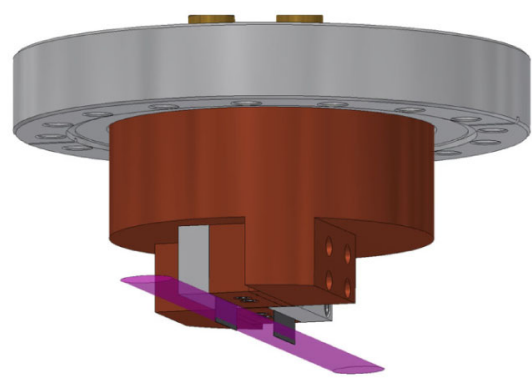

(a)

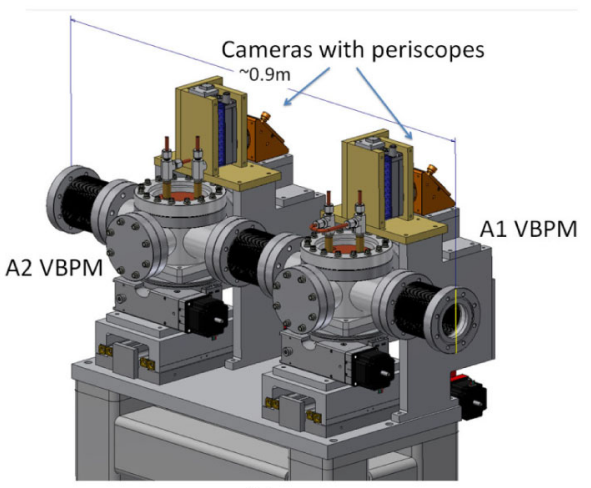

(b)

FIG. 24. vBPM developed for A-line. (a) Schematic of diamond blades intercepting synchrotron radiation. The blades are separated by approximately $7 \mathrm{~mm}$; (b) Implementation of two adjacent vBPMs for monitoring positions of two canted undulator beams separated by $10 \mathrm{~mm}$ ( $1 \mathrm{mrad}$ angular separation). 
level outputs are connected to $200 \mathrm{~W}, 150 \mathrm{MHz}$ ENI amplifiers which drive horizontal and vertical stripline kickers. The longitudinal processor drives a $200 \mathrm{~W}$ solid-state RF amplifier that in turn drives a $1 \mathrm{GHz}$ low Q cavity kicker. While the system can handle bunch spacings as short as $2 \mathrm{~ns}$, gain falls off because of the short interval required to provide feedback. A bunch spacing of $14 \mathrm{~ns}$ provides for the maximum kick as well as matching better with the injector RF frequency.

\section{BUNCH PATTERNS}

Historically, bunch patterns were constrained by the beam-beam interaction from electrostatically-separated counter-rotating beams in the same vacuum chamber. With the simplification of single-beam operation, there are many more possibilities.

There is increasing interest in time-resolved experiments (see, for example, $[25,26]$ ), and a number of possible bunch patterns are under consideration. The constraints on bunch patterns are now discussed, and several bunch patterns proposed.

\section{A. Mode coupling instability threshold}

Many light source upgrades are favoring multibend achromat designs with strong focusing and small vacuum apertures (see, for example, [27,28]). However, the resistive wall impedance scales as $1 / r^{3}$, quickly limiting the singlebunch current due to mode coupling instability [29]. The vacuum chamber aperture for CHESS-U has been kept relatively large $(52 \mathrm{~mm} \times 22.5 \mathrm{~mm}$ full-aperture $)$ in an effort to keep the maximum single bunch current as high as possible.

The transverse mode coupling instability (TMCI) threshold has been estimated for CHESS-U based on analytic resistive wall calculations [30] and detailed T3P modeling for discontinuities [31,32]. The limit is estimated to be around $25 \mathrm{~mA}$ (64 nC) [33]; conservatively, only bunch patterns up to $20 \mathrm{~mA}$ are considered.

The present single-bunch current limit is $10 \mathrm{~mA}(26 \mathrm{nC})$ per bunch, to protect instrumentation (BPM modules and feedback amplifiers). Options for raising the limit are under discussion, opening the path to high bunch-current operating modes. These options include preventing damage to the feedback amplifiers by limiting beam-induced power transmitted back to the amplifier.

\section{B. Momentum aperture and Touschek lifetime}

The momentum compaction factor $\alpha_{p}$ for CHESS-U is rather large compared to other modern light sources, resulting in a limited momentum aperture (Fig. 25, top). The Touschek lifetime (Fig. 25, bottom) at the design emittance coupling of $1 \%$, total RF voltage of $6 \mathrm{MV}$, and design bunch current of $2.22 \mathrm{~mA}$ (in $18 \times 5$ mode) is roughly $40 \mathrm{hrs}$.

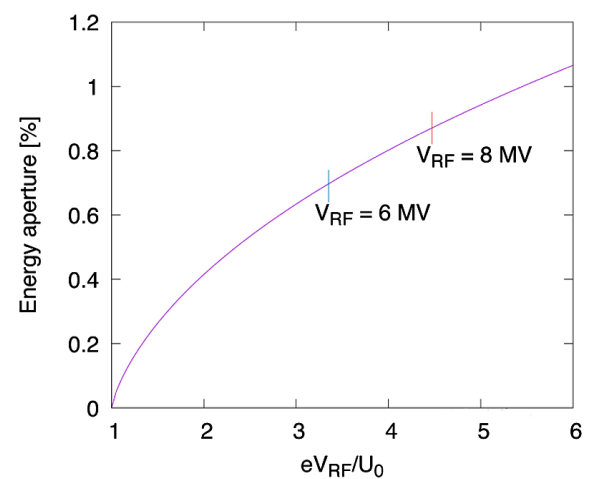

(a)

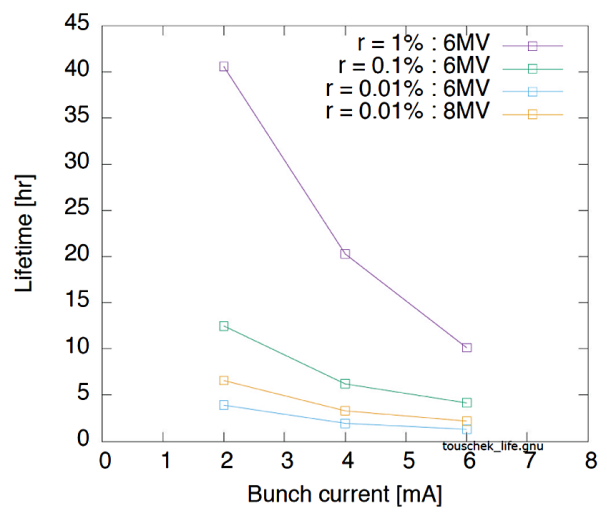

(b)

FIG. 25. Momentum aperture and Touschek lifetime as a function of rf voltage, bunch current, and emittance coupling. (a) Momentum aperture; (b) Touschek lifetime.

Extrapolating to $25 \mathrm{~mA}$, the anticipated Touschek lifetime at $0.1 \%$ coupling is approximately one hour; at $1 \%$ coupling, this increases to around 3 hours.

\section{Electron cloud}

Initial commissioning will be done with positrons. As such, bunch patterns will be limited by tune shift and head-tail instability due to the electron cloud effect [34]. Each of the bunch patterns proposed here were examined for electron cloud build-up and trapping [35]. The electron cloud tune shift was constrained to be less than or equal to the electron cloud tune shift in present two-beam bunch patterns (around $3.5 \mathrm{kHz}$, or $\Delta Q \approx$ 0.009 in both horizontal and vertical). It is possible that larger tune shifts from electron cloud would be tolerable.

\section{Proposed bunch patterns}

Bunch patterns proposed for CHESS-U are listed in Table IV. Commissioning and early operation will be in the $18 \times 5$ pattern, with the option of exploring other bunch patterns as user interest dictates. 
TABLE IV. Bunch patterns for CHESS-U. $1 \mathrm{~mA}=1.6 \times 10^{10}$ particles $=2.56 \mathrm{nC}$.

\begin{tabular}{lccc}
\hline \hline Parameter & $18 \times 5$ & $9 \times 5$ & $9 \times 1$ \\
\hline No. Trains & 18 & 9 & 9 \\
Train Spacing [ns] & 84 & 224 & 280 \\
No. Bunches/Train & 5 & 5 & 1 \\
Bunch Spacing [ns] & 14 & 14 & \\
Charge/Bunch [mA] & 2.22 & 4.44 & 20 \\
Total current [mA] & 200 & 200 & 180 \\
$\tau_{\text {Touschek at } 1 \% \text { Coupling [hrs] }}$ & 40 & 17 & 4 \\
\hline \hline
\end{tabular}

In any of the proposed bunch patterns, CESR would run with topoff. In present CHESS two-beam conditions, the topoff interval for positrons is $5 \mathrm{~min}$, with a Touschek lifetime of approximately $12 \mathrm{hrs}$.

CESR will preserve the ability to store electrons in the counter-clockwise direction for machine studies. Total current in the counterclockwise direction will be limited to $5 \mathrm{~mA}(13 \mathrm{nC})$ at $6 \mathrm{GeV}$, limited by synchrotron radiation heating on the sliding joints in the new achromats. This current limit will scale as $E^{-4}$; therefore, the restriction in the counterclockwise direction at $2.1 \mathrm{GeV}$ (frequently used for machine studies) will exceed the existing administrative limit of $200 \mathrm{~mA}$.

\section{INSTALLATION AND COMMISSIONING}

Installation of the CHESS-U upgrade began in June 2018, and is scheduled to be completed in November 2018. Beam commissioning will follow. All insertion devices are out-of-vacuum, which allows for initial commissioning and vacuum processing without IDs. Undulators will be installed one canted pair at a time, with a brief recovery period after each installation to establish operating conditions. Beam will be delivered to all end stations by April 2019.

The positron beam presently circulates in the clockwise direction; as such, initial commissioning and operation of CHESS-U will be with positrons, with the option to invert the polarity of the injector and storage ring to run electrons in the clockwise direction at a future date.

\section{SUMMARY}

CHESS-U will deliver high flux photon beams at high energy, enabling a diverse set of new experiments [12]. The upgrade will improve the performance of the CESR storage ring for $\mathrm{x}$-ray production by almost an order of magnitude with CCUs and a factor of three for the 24-pole "F-line" wiggler. In turn, the transition to single-beam will drastically simplify operations. Bunch patterns compatible with timing mode are under consideration, pending evaluation during commissioning and machine studies.

\section{ACKNOWLEDGMENTS}

The authors wish to thank everyone involved in the CHESS-U upgrade project, and those who have contributed material to this paper. Funding for the CHESS-U upgrade provided by New York State Capital Grant No. AA737/ CFA and No. 53676.

\section{APPENDIX A: MISALIGNMENTS FOR ERROR TOLERANCE}

Standard deviations for misalignments applied in error tolerance simulations are listed in Table V. The errors for dipoles, quadrupoles, and sextupoles are based on survey information. DQ and girder errors are determined by extrapolation from survey information and magnetic alignment characterization.

BPM misalignments and errors are listed in Table VI.

TABLE VI. RMS BPM errors for misalignment and correction simulation.

\begin{tabular}{lc}
\hline \hline Error & Amplitude \\
\hline Transverse offset $[\mu \mathrm{m}]$ & 170 \\
Reproducibility $[\mu \mathrm{m}]$ & 10 \\
Roll [mrad] & 12 \\
Timing $[\mathrm{ps}]$ & 10 \\
Shear $[\mu \mathrm{m}]$ & 100 \\
Channel gain $[\%]$ & 0.5 \\
\hline \hline
\end{tabular}

TABLE V. RMS error amplitudes for misalignment and correction simulation.

\begin{tabular}{lccccc}
\hline \hline Error & Dipole & DQ & Quad & Sext & Girder \\
\hline X-offset $[\mu \mathrm{m}]$ & 2000 & 100 & 200 & 300 & 100 \\
y-offset $[\mu \mathrm{m}]$ & 900 & 100 & 110 & 300 & 100 \\
S-offset $[\mathrm{mm}]$ & 2.3 & 2.0 & 5.2 & 5.2 & 0.1 \\
Pitch $[\mu \mathrm{rad}]$ & 600 & 87 & 1100 & 1200 & 100 \\
Yaw $[\mu \mathrm{rad}]$ & 300 & 87 & 62 & 800 & 100 \\
Roll $[\mu \mathrm{rad}]$ & 500 & 500 & 500 & 500 & 100 \\
Gradient $[\%]$ & & 0.1 & 0.1 & 0.1 & \\
\hline \hline
\end{tabular}


TABLE VII. Systematic multipoles used in tracking simulations. "CESR" magnets are those which are outside the new CHESS-U achromats. Evaluated as $d B / B$ at a reference radius of $20 \mathrm{~mm}$.

\begin{tabular}{|c|c|c|}
\hline Element Class & Multipole & Amplitude \\
\hline CESR Dipole & $\begin{array}{l}b_{1} \\
b_{2} \\
b_{12}\end{array}$ & $\begin{array}{l}-0.5 \times 10^{-4} \\
-0.2 \times 10^{-4} \\
-2.4 \times 10^{-9}\end{array}$ \\
\hline CESR Quadrupole & $\begin{array}{l}b_{5} \\
b_{9}\end{array}$ & $\begin{array}{r}1.5 \times 10^{-4} \\
-0.053 \times 10^{-4}\end{array}$ \\
\hline CESR Sextupole & $b_{8}$ & $1 \times 10^{-4}$ \\
\hline CHESS-U Quads A/D & $\begin{array}{l}b_{2} \\
b_{3} \\
b_{4} \\
b_{5} \\
a_{2} \\
a_{3} \\
a_{4} \\
a_{5}\end{array}$ & $\begin{array}{r}1 \pm 6.5 \times 10^{-4} \\
0.4 \pm 1.7 \times 10^{-4} \\
-0.3 \pm 1.5 \times 10^{-4} \\
-4 \pm 1.5 \times 10^{-4} \\
0.8 \pm 7.8 \times 10^{-4} \\
-0.3 \pm 2.8 \times 10^{-4} \\
-0.5 \pm 1.9 \times 10^{-4} \\
0.1 \pm 0.6 \times 10^{-4}\end{array}$ \\
\hline CHESS-U Quads B/C & $\begin{array}{l}b_{2} \\
b_{3} \\
b_{4} \\
b_{5} \\
a_{2} \\
a_{3} \\
a_{4} \\
a_{5}\end{array}$ & $\begin{array}{r}1.2 \pm 6.1 \times 10^{-4} \\
0.8 \pm 2.3 \times 10^{-4} \\
0.8 \pm 1.8 \times 10^{-4} \\
-2.6 \pm 2.1 \times 10^{-4} \\
-1.7 \pm 3.3 \times 10^{-4} \\
-2.6 \pm 4.0 \times 10^{-4} \\
-2.2 \pm 2.9 \times 10^{-4} \\
0.3 \pm 0.7 \times 10^{-4}\end{array}$ \\
\hline CHESS-U DQ & $\begin{array}{l}b_{2} \\
b_{3} \\
a_{1} \\
a_{2} \\
a_{3}\end{array}$ & $\begin{array}{r}-3.4 \pm 5.6 \times 10^{-4} \\
-2.3 \pm 1.9 \times 10^{-4} \\
-1 \pm 2.8 \times 10^{-4} \\
-0.6 \pm 2.6 \times 10^{-4} \\
0.1 \pm 1.4 \times 10^{-4}\end{array}$ \\
\hline
\end{tabular}

\section{APPENDIX B: SYSTEMATIC MULTIPOLES}

The multipole expansion is parametrized as a fractional field error $d B / B$ with normal $\left(b_{n}\right)$ and skew $\left(a_{n}\right)$ components, where $n=1$ corresponds to a quadrupolelike field. The reference radius for all multipoles listed in Table VII is $20 \mathrm{~mm}$. All multipoles are normalized to the main field.

[1] M. Billing, The conversion of CESR to operate as the Test Accelerator, CesrTA. Part 1: overview, J. Instrum. 10, P07012 (2015).

[2] S. Wang, D. L. Rubin, and J. P. Shanks, CESR Lattice for Two Beam Operations with Narrow Gap Undulators at CHESS, in Proceedings of International Particle Accelerator Conference (IPAC'16), Busan, Korea, 2016 (2016), pp. 2968-2971, http://accelconf.web.cern.ch/accelconf/ ipac2016/papers/wepow053.pdf.
[3] D. Sagan and J. C. Smith, The TAO accelerator simulation program, in Proceedings of the 2005 Particle Accelerator Conference, Knoxville, TN, edited by C. Horak (IEEE, New York, 2005), pp. 4159-4161.

[4] D. Sagan, Bmad: A relativistic charged particle simulation library, Nucl. Instrum. Methods Phys. Res., Sect. A 558, 356 (2006).

[5] A. Mikhailichenko, Optimized Wiggler magnet for CESR, in Proceedings of the 19th Particle Accelerator Conference, Chicago, IL, 2001 (IEEE, Piscataway, NJ, 2001), pp. 3648-3650.

[6] J. Bengtsson, The sextupole scheme for the Swiss Light Source (SLS): An analytic approach, Paul Scherrer Institut Technical Report No. SLS-TME-TA-1997-0009, 1997.

[7] A. Temnykh, D. Dale, E. Fontes, Y. Li, A. Lyndaker, P. Revesz, D. Rice, and A. Woll, Compact undulator for the Cornell High Energy Synchrotron Source: Design and beam test results, J. Phys. Conf. Ser. 425, 032004 (2013).

[8] A. Temnykh, A. Lyndaker, M. Kokole, T. Milharcic, J. Pockar, and R. Geometrante, Construction of CHESS compact undulator magnets at Kyma, in Proceedings of Advances in X-ray Free-Electron Lasers Instrumentation III (SPIE, Prague, Czech Republic, 2015), Vol. 9512, https://doi .org/10.1117/12.2186301.

[9] A. Temnykh, T. Kobela, A. Lyndaker, J. Savino, E. Suttner, and Y. Li, Compact undulator for Cornell High Energy Synchrotron Source, IEEE Trans. Appl. Supercond. 22, 4100504 (2012).

[10] K. Finkelstein, The new CHESS wiggler, Rev. Sci. Instrum. 63, 305 (1992).

[11] T. Tanaka and H. Kitamura, Spectra: A synchrotron radiation calculation code, J. Synchrotron Radiat. 8, 1221 (2001).

[12] J. Brock and E. Fontes, New science made possible by CHESS-U, CLASSE, Cornell University Technical Report, 2016.

[13] J. Shanks, D. L. Rubin, and D. Sagan, Low-emittance tuning at the Cornell Electron Storage Ring Test Accelerator, Phys. Rev. ST Accel. Beams 17, 044003 (2014).

[14] D. Sagan, Betatron phase and coupling correction at the Cornell Electron/positron Storage Ring, Phys. Rev. ST Accel. Beams 3, 102801 (2000).

[15] J. Laskar, Frequency analysis for multi-dimensional systems. global dynamics and diffusion, Physica D: Nonlinear Phenomena 67, 257 (1993).

[16] A. Temnykh, CHESS-U external review, Cornell University Technical Report, 2016.

[17] R. Littauer, S. E. Ball, R. G. Helmke, S. B. Peck, and D. H. Rice, The cesr control system, IEEE Trans. Nucl. Sci. 26, 3275 (1979).

[18] M. J. Forster, S. Ball, L. Bartnik, D. Bougie, R. Helmke, M. Palmer, S. Peck, D. Riley, R. Sholtys, and C. Strohman, CESR control system upgrade to linux high availability cluster, in Proceedings of the 2012 International Particle Accelerator Conference, New Orleans, LA (IEEE, Piscataway, NJ, 2012), pp. 3999-4001.

[19] A. Temnykh, Vibrating wire field-measuring technique, Nucl. Instrum. Methods Phys. Res., Sect. A 399, 185 (1997).

[20] M. G. Billing, J. A. Dobbins, M. J. Forster, D. L. Kreinick, R. E. Meller, M. C. Rendina, N. T. Rider, D. C. Sagan, J. Shanks, J. P. Sikora, M. G. Stedinger, C. R. Strohman, 
H. A. Williams, M. A. Palmer, R. L. Holtzapple, and J. Flanagan, Instrumentation for the study of low emittance tuning and beam dynamics at CESR, J. Instrum. 12, T11006 (2017).

[21] M. G. Billing, W. F. Bergan, M. J. Forster, R. E. Meller, M. C. Rendina, N. T. Rider, D. C. Sagan, J. Shanks, J. P. Sikora, M. G. Stedinger, C. R. Strohman, M. A. Palmer, and R. L. Holtzapple, Beam position monitoring system at CESR, J. Instrum. 12, T09005 (2017).

[22] A. Temnykh, D. Dale, E. Fontes, A. Lyndaker, Y. Li, J. Ruff, P. Revesz, and A. Woll, Chess upgrade with compact undulator magnets: Operating experience and first results, AIP Conf. Proc. 1741, 020003 (2016).

[23] P. Revesz, A. Lyndaker, A. Pauling, and A. Temnykh, Novel video beam position monitor for canted undulators at CHESS, CHESS E-News, 2015.

[24] D. Teytelman, iGp-1281F signal processor: Technical user manual, revision 1.7, Dimtel, Inc. Technical Report, 2009.

[25] P. K. Lambert, C. J. Hustedt, K. S. Vecchio, E. L. Huskins, D. T. Casem, S. M. Gruner, M. W. Tate, H. T. Philipp, A. R. Woll, P. Purohit, J. T. Weiss, V. Kannan, K. T. Ramesh, P. Kenesei, J. S. Okasinski, J. Almer, M. Zhao, A. G. Ananiadis, and T. C. Hufnagel, Time-resolved x-ray diffraction techniques for bulk polycrystalline materials under dynamic loading, Rev. Sci. Instrum. 85, 093901 (2014).

[26] J. Liu, N. Dauphas, M. Roskosz, M. Y. Hu, H. Yang, W. Bi, J. Zhao, E. E. Alp, J. Y. Hu, and J.-F. Lin, Iron isotopic fractionation between silicate mantle and metallic core at high pressure, Nat. Commun. 8, 14377 (2017).
[27] APS-U preliminary design report, Argonne National Laboratory Technical Report, 2012.

[28] R. Dimper, H. Reichert, P. Raimondi, L. S. Ortiz, F. Sette, and J. Susini, ESRF upgrade programme phase II technical design study, European Synchrotron Radiation Facility Technical Report, 2015.

[29] F. J. Sacherer, Bunch lengthening and microwave instability, IEEE Trans. Nucl. Sci. 24, 1393 (1977).

[30] R. L. Gluckstern, J. van Zeijts, and B. Zotter, Coupling impedance of beam pipes of general cross section, Phys. Rev. E 47, 656-663 (1993).

[31] Advanced Computational Electromagnetics 3P Code Suite (SLAC, Stanford, CA), https://confluence.slac.stanford.edu/ display/AdvComp/ACE3P+-+Advanced+Computational+ Electromagnetic+Simulation+Suite.

[32] L. Lee, V. Akcelik, L. Ge, S. Chen, G. Schussman, A. Candel, Z. Li, L. Xiao, A. Kabel, R. Uplenchwar, C. Ng, and K. Ko, Computational science research in support of petascale electromagnetic modeling, J. Phys. Conf. Ser. 125, 012077 (2008).

[33] L. Salo, Estimating the longitudinal and transverse intensity-dependent behavior of CESR, REU Report (CLASSE, Cornell University, Ithaca, NY, 2017).

[34] J. R. Calvey, Studies of electron cloud growth and mitigation at Cesr-TA, Ph. D. thesis, Cornell University, Ithaca, New York, 2013.

[35] K. Rowan, Revised predictions of electron-cloud-based instabilities for CHESS-U, REU Report (CLASSE, Cornell University, Ithaca, NY, 2018). 